\title{
Genome-wide Identification, Characterization and Expression profile of TALE gene family in Juglans regia $L$.
}

\author{
Caihua Guo \\ Shihezi University College of Agriculture \\ Jianxin Niu ( $\square$ njx105@163.com ) \\ Shihezi University \\ Shaowen Quan \\ Shihezi University College of Agriculture \\ Zhongrong Zhang \\ Shihezi University College of Agriculture \\ Chao Kang \\ Shihezi University College of Agriculture \\ Jinming Liu \\ Shihezi University College of Agriculture
}

Research article

Keywords: Walnut (Juglans regia), Genome-wide identification, Phylogenetic analysis, Conserved motif, Promoter Cis-acting elements, Expression profiles

Posted Date: August 1st, 2020

DOI: https://doi.org/10.21203/rs.3.rs-41834/v1

License: (c) (i) This work is licensed under a Creative Commons Attribution 4.0 International License. Read Full License

Version of Record: A version of this preprint was published at Scientia Horticulturae on April 1st, 2022. See the published version at https://doi.org/10.1016/j.scienta.2022.110945. 


\section{Abstract \\ Background}

The three amino acid loop extension (TALE) are essential transcription factors that regulate the differentiation of meristem in the plant growth and development. there's a lot of journalism of the TALE family in plants, such as Arabidopsis, rice, and others. however, there's no report on the TALE family in walnut.

\section{Results}

In this study, the genome-wide identification and expression analysis of the TALE gene family were studied comprehensively in walnut. Based on the specific structure of the TALE domain, a total of twenty-seven walnut TALE genes (JrTALE) were identified. According to different domains structure and phylogenetic relationships, the JrTALE gene family could be divided into two subfamilies, namely BELL and KNOX. This family was relatively conservative in evolution. Exon-intron structure analysis showed that there were 3 to 6 exons, and they had a wide range of exons length. Analysis of protein conserved motifs showed that JTTALE contained similar motif structures in the same phylogenetic branch. Expression pattern analysis of the TALE family genes in walnut showed that JrTALE41 and JrTALE42 were differentially expressed in female and male flower buds. We confirmed that JrTALE4-5, JrTALE8-12, JrTALE36-37, JrTALE46-53, and JrTALE14-15 were differentially expressed between different stages of flower bud development by real-time quantitative PCR (qRT-PCR).

\section{Conclusions}

Sixty-nine JTTALEs were identified in the genome-wide. the physicochemical properties, phylogenetic relationships, gene structure, promoter cis-acting elements, and protein interactions were systematically analyzed by bioinformatics methods. This study provides valuable information about the TALE family in the transitional development of walnut flower buds, which lay the foundation for further understanding of TALE family genes and in walnut.

\section{Background}

The Three Amino acid Loop Extension (TALE) transcription factors are widely present in animals, plants, and other eukaryotes, which play important roles in the plant meristem development and other important plant biological processes [1]. Gehring [2] and other scholars isolated an extremely conserved sequence in which some genes were mutated to produce a homologous phenotype, which was later called the homeobox. The family gene contains KNOTTED-like homeodomain (KNOX) and BEL1-like homeodomain (BLH / BELL) proteins [3], which function as heterodimers that are structurally and functionally related. The TALE family gene encodes an atypical homeobox domain containing 63 amino acid residues. There is a three-amino acid extension in the loop connecting the first and second helices of their homeodomain, so the family is called homeobox protein superfamily [4-5]. Therefore, the TALE transcription factors of Arabidopsis thaliana are divided into BELL subfamily and KNOX subfamily [5]. At the same time, these two subfamilies have specific interactions [6], which can regulate downstream genes to achieve different biological functions. KNOX subfamily is almost distributed in plants [7], and it has four domains, namely KNOX1, KNOX2, ELK, and Homeobox KN [8-9]. The maize Knotted-1 (Kn1) gene is the first homeobox gene found in plants [10]. Recessive mutations in the SHOOTMERISTEMLESS (STM) gene have resulted in the inability of the stem-tip meristem (SAM) to form or be maintained in Arabidopsis [11-12]; KNAT7 is involved in the secondary cell wall biosynthesis [13]; GhKNL1, a member of the TALE family in cotton, regulated the synthesis of cotton fibers [14]; in the TALE family, SHOOTMERISTEEMLESS (STM), KNAT2, BREVIPEDICELLUS (BP) / KNAT1, and KNAT6 genes in the KNOX Class I family in Arabidopsis mainly regulate the apical meristem, leaf

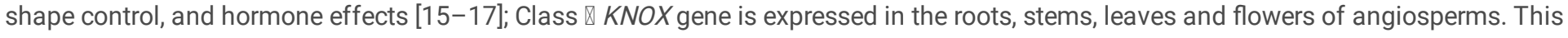
group of genes is mainly involved in regulating the differentiation of plant organs [18-20]; KNOX proteins are also involved in regulating the anabolic metabolism of many substances, including gibberellic acid (GA), cytokinin (CK), and lignin [21-22]. The BELL subfamily has four domains in rice, namely SKY, BEL, Homeobox KN, and VSLTLGL (ZIBEL) [23-24]. Members of the BELL family also have important regulatory roles in the development and transformation of flowers. Overexpression of BLH6 gene delays flowering, and overexpression of the BLH3 gene makes flowering earlier [25]. Different KNOX and BELL selectively combine to form KNOX-BELL heterodimers heteromers, which specifically bind to specific target sequences. It can be concluded that different combinations of two subfamilies can regulate different downstream genes to complete their functions in plants $[6,26]$. The dimer of BELL and KNOX StBEL5 / POTH1 can inhibit the expression of the key enzyme ga20ox1 gene in the biochemical pathway of GA [27]. Members of the KNOX family, STM, and members of the BELL family, ATH1 and PENNYWISE (PNY), have similar functions and regulate the development of plant meristems [28-29]. In 
Arabidopsis, BLH1 and KNAT3 proteins form heterodimers, which regulate the expression level of ABA-responsive genes and affect seed germination and growth [30]; KNAT1 interacts with RPL, FUL and AP to regulate the development of pistil marginal tissue [31-32].

Walnut is an important economic tree species cultivated worldwide with its high-quality woody oils and nuts [33]. It belongs to a hermaphrodite, flowers are the most complicated organs in plants. The formation of flower buds means the beginning of reproductive growth, so flowering biology has always been a research hotspot in the field of plants. According to previous research, some members of the TALE family play an important role in meristem development $[15,34]$. In order to better understand the molecular mechanism of walnut flower bud transition, it is necessary to research the TALE gene family in walnut. There are few reports on the role of the TALE gene family in walnuts. In this study, members of JrTALE gene family were identified in the genome-wide. The physicochemical properties, phylogenetic relationships, gene structure, gene duplication, and protein interactions were systematically analyzed by bioinformatics methods.

Transcriptome data were used to explore the ex-pression patterns of the JrTALE gene family in different tissues (male and female flower buds) and different development stages, and verified by qRT-PCR. This study lay the foundation for further exploring the regulatory role of the walnut TALE gene.

\section{Results}

\section{Genome-wide identification of TALE family members in walnut}

Sixty-nine TALE gene family members were identified from the walnut genome sequence, which were encoded by twenty-seven genes and located on twenty-five scaffolds. As can be seen from Table 1, the length of the encoded amino acid sequence is between 251-550aa, of which JrTALE55 encodes the shortest amino acid, and JrTALE16-JITALE22 has the longest; the molecular weight of the protein is 28666.793611.11 Da, and the isoelectric point is 4.87-9.77. ProtComp 9.0 online software was used to perform subcellular localization analysis on the predicted protein. The results showed that JrTALE was located in the nucleus. As shown in Additional file 2: Table S1.

\section{Phylogenetic analysis of TALE gene family}

Based on MUSCLE multiple sequence alignment, the MEGA6.0 software was used to construct a neighbor-joining (NJ) phylogenetic tree for the amino acid sequences of 69 walnuts TALE proteins, 33 Arabidopsis TALE proteins, and 21 grape TALE proteins (Fig. 1). Based on the phylogenetic tree and previous studies, all members of the TALE gene family were divided into two subfamilies KNOX and BELL according to different domains. The distribution of JrTALE proteins among different subfamilies was as following: KNOX (14) and BELL (55), In each evolutionary branch, there were multiple branches from the same species, which may be caused by gene replication events, and the lack of representatives of certain species may be related to gene loss events in the family. In the KNOX subfamily, JrTALE35 and grape GSVIVT01007715001 were orthologous genes; In the BELL subfamily, GSVIVT01009779001 and JrTALE40 were orthologous genes.

\section{Exon-intro Structure Analysis of TALE family members in walnut}

The gene structure of JrTALE family members was analyzed online by GSDS web server (Fig. 2). The results showed that there were evident differences in the number of exons among all family members, with a maximum of 6 exons. Only JrTALE55 contains 3 exons; most family members contained four exons. JrTALE55, JTTALE26, JrTALE27, JrTALE36, and JrTALE37 contained 3' UTR (untranslated regions), which helped to bind microRNAs and regulate the expression of transcription factor proteins. the gene structure was combined with the clustering of the phylogenetic tree. the differences in gene structure between each subfamily were small, the result indicated that the structural similarity of the same subfamily was high.

\section{Conserved motifs analyses}

Conservative motif analysis can reveal the diversity of TALE protein and its functional differentiation in walnuts. family members containing the same motif may have similar functions; family members contained different motif elements suggesting that may have undergone functional differentiation and have other special functions. the MEME online tool was used to predict the conserved motifs of walnut TALE family members (Fig. 3). All JITALE members contained motif 1 , the results showed that it was highly conserved in the TALE gene family; motif 9 was highly conserved at the N-terminus in the KNOX subfamily. Except JrTALE55, all BELL subfamily members had seven common conserved motifs were motif8, motif5, motif2, motif4, motif3, motif1, motif7, respectively. The phylogenetic tree shows that closely related members of the TALE family contain the same conserved elements, however, the same subfamily has a proprietary conserved motif, indicating that the same subfamily has similar functions.

\section{Cis-elements prediction in the promoter of JrTALE gene}


In response to plant hormones, stress factors and light response, the cis element in the promoter region affects gene expression to regulate plant development and adaptability to environmental changes. In order to obtain more information about the function of the TALE gene in walnuts, 26 JrTALE gene promoters were analyzed using the online software PlantCARE (of which the JrTALE55sequence was too short to be analyzed). The cis-elements were divided into four main subgroups: stress-responsive, hormone-responsive, light-responsive, and MYB binding sites (Fig.4). Most genes were involved in the light-response type promoter (52.51\%), followed by hormones (27.41\%) and other stress-related genes (13.51\%). Among 26 JrTALES, eleven of them possesed MYB binding sites. In addition, we found that there were abundant light-responsive cis-elements in the JrTALES, especially in the JrTALE41 gene with a number of 19.

\section{Gene Expression Analysis}

Based on RNA-seq data, we used the FPKM values of $27 \mathrm{JrTALE}$ genes to investigate the expression profile of JrTALE family genes. (i) Comparison of JrTALE gene expression levels in female flower buds and leaf buds (Fig. 5A). JrTALE36-37and JrTALE46-53 were highly expressed in female and male flower buds, JrTALE29-31, JrTALE55, JrTALE32-33, and JrTALE54 were lowly expressed in female and male flower buds, and the expression levels of JrTALE41 and JrTALE42 in female flower buds were higher than male flower buds. (ii) The expression levels of JrTALE gene before, during, and after transformation of female flower buds (FB-1 / FB-2 / FB-3) and before, during, and after transformation of male flower buds (MB-1/ MB-2 / MB-3) were compared (Fig. 5B). JrTALE55 was not expressed in FB; JrTALE812, JrTALE46-53 and JrTALE14-15 were highly expressed in FB-1 and FB-3; JrTALE23-25, JrTALE55, JrTALE29-31 were lowly expressed in female and male flower buds; JrTALE14-15, JrTALE41 and JrTALE42 were differentially expressed in FB-1, FB-2, FB-3, MB-1, MB-2, MB-3.

\section{Verification of JITALE expression pattern by qRT-PCR}

Based on this data comes from the analysis results of JrTALE expression pattern, six genes (JrTALE4-5, JrTALE8-12, JrTALE14-15, JrTALE23-25, JrTALE36-37, JrTALE46-53) were further analyzed to detect their expression patterns in different tissues and different development stages using qRT-PCR (Fig. 6). Of these, the expression levels of JrTALE4-5, JrTALE23-25, JrTALE36-37 and JrTALE46-53 were the highest in the male flower buds, consistent with those of the RNA-seq data; As for flower bud transition periods, three genes (JrTALE4-5, JrTALE36-37 and JrTALE46-53) were up-regulated in FB / MB-3 than that in FB / MB-2; Moreover, At different developmental stages, the expression of JrTALE14-15 in female flower buds was higher than that of male flower buds; The suggest that this gene should work as the candidate gene for female and male flower buds difference expression in walnut (Fig. 6).

\section{Protein interaction network of the JrTALE family}

The function of Arabidopsis TALE has been studied in depth, but the relationship of walnut TALE protein interaction has not been reported. Therefore, we can construct a walnut TALE protein interaction network through the interaction of Arabidopsis TALE protein. twenty-one homologous proteins and five predicted functional proteins were obtained, and the walnut TALE protein corresponding to the Arabidopsis TALE protein was listed below it (Fig. 7). It can be seen from the figure that several JrTALE genes (For example, the homologous Arabidopsis BLH2 and KNAT6 corresponding to the walnut TALE proteins) were the core nodes for predicting protein interactions. this result suggests that they may participate in multiple functions by interacting with other proteins.

\section{Discussion}

TALE transcription factor is an important transcription factor regulating plant growth and development, meristem maintenance and organ morphogenesis [21, 34, 35]. The TALE gene family included three domains, namely KNOX, ELK and Homeobox. the ELK domain was an amphoteric helix structure, which played an important role in protein-protein interactions; the Homeobox domain was used for binding to target genes. So far, little is known about the regulatory role of the TALE gene family in the development of female and male flower buds in walnut.

\section{Analysis of Phylogenetic and gene structure}

Analysis of the physicochemical properties of proteins showed that the protein molecular mass and amino acid quantity of different members of JrTALE family were quite different (Table 1), indicating that the family may have complex regulatory modes. It was reported that the MEINOX domain upstream of the KNOX protein homology domain combined with the BELL domain upstream of the BELL protein homology domain to form a KNOX / BELL heterodimer, which was transported from the cytoplasm to the nucleus to play a role in regulate all aspects of plant development [36]. The results of subcellular localization (Additional file 2: Table S1) in the nucleus were consistent with previous studies. Phylogenetic analysis divided the TALE family into BELL and KNOX subfamilies based on evolutionary relationships, and its branches were more obvious, indicating that the functions of these two subfamilies were different and had long been differentiated 
(Fig. 1). However, only 5 of the 69 JrTALE family members contained a 3 ' UTR (Fig. 2). This structure served as a possible binding site for miRNA ${ }^{[21]}$, indicating that it had more abundant regulatory characteristics for downstream genes. All members of the JrTALE family contained a motif 1 (Homobox conserved domain) (Additional file 1: Figure S1 and Fig. 3), and the distribution of motif was highly conserved, which provided a reliable targeting sequence for the following functional verification studies.

\section{Expression analysis of JrTALE gene}

Gene differential expression analysis identified that the expression levels of JrTALE14-15, JrTALE16-22, JrTALE41 and JrTALE42 in female flower buds were higher than those in male flower buds, which indicated that these JrTALE genes may positively regulate the development of female flower buds. Before the flower bud transition (FB / MB-1) compared with during (FB / MB-2) and after (FB / MB-3) transformation of flower buds, the three JrTALE genes (JrTALE36-37, JrTALE46-53, JrTALE8-12) were highly expressed before the flower bud transition (FB / MB-1), and expressed at different developmental stages of flower buds, indicating that these JrTALE genes may actively participate in the regulation of walnut floral organ development. The KNOX subfamily was involved in the maintenance of meristems and the occurrence of organ-specific patterns. The JrTALE genes (JrTALE41 and JrTALE42) differentially expressed in the KNOX subfamily were only expressed in the female flower buds, indicating that these JrTALE genes may be involved in the development of pistil, This result consistent with the results of the Arabidopsis study [37-38]. The $B L H 8$ / PNF gene in Arabidopsis was mainly involved in meristem growth maintenance and flowering signal transduction [39-40]. The JrTALE gene (JrTALE16-22) was identified as BLH8 (as shown in Fig. 7) and was divided into BELL subfamilies, And the expression level in FB-3 was higher than FB-1 and FB-2, the results show that the JrTALE gene is involved in the growth and maintenance of meristematic tissues.

\section{Analysis of JrTALE protein-protein interaction}

The BELL protein and the KNOX protein specifically recognized and binded to form a heterodimer, and the Homeobox domains of these two proteins recognizeed and specifically binded to their respective target gene sequences, so TALE gene family may regulate the expression level of downstream genes $[21,36]$. So far, the interaction network of the TALE gene family in Arabidopsis has been extensively studied. JrTALE8-12 / JrTALE46-53 / JrTALE26 were homologous to BLH1 / KNAT3. In Arabidopsis, the BLH1 protein of the BELL subfamily can combine with the KNAT3 protein of the KNOX subfamily to form BLH1-KNAT3 dimers, which affected the germination and growth of seeds by regulating the expression of genes related to the ABA signaling pathway [41-42]. JrTALE43-44 / JrTALE59-69 / JrTALE6-7 / JrTALE34 / JrTALE41 / JrTALE45 / JrTALE35 were homologous to ATH1 / STM / KNAT2, and the dimer formed by the ATH1 protein of the BELL subfamily and the STM protein of the KNOX subfamily can regulate the development of the meristem at the top of the plant [45], while the dimer formed by the ATH1 protein and the KNAT2 protein regulated the development of the inflorescence of the plant [44]. AS1 may cooperate with AS2 to inhibit the expression of KNOX $\otimes$ (including STM, KNAT1 / BP, KNAT2, KNAT6) in the heart flap and placenta frame [45-46]. In addition, as the core node of the interaction network, JrTALEs (JrTALE28, JrTALE1 and JrTALE2) and JrTALE protein interaction network also had many interaction partners (Fig. 7), these results illustrated how JrTALE family proteins form functional complexes that mediate the expression of genes at different developmental stages of male and female flower buds in walnut.

\section{Conclusions}

In this study, 69 JrTALE were identified in walnut genome and were classified into two subfamilies. Members of the same subfamily generally had conserved gene structure and protein motif composition. BELL family proteins and KNOX family proteins perform specific binding to form heterodimers and regulate all aspects of plant development. Moreover,many JrTALE genes had relatively high expression levels in the female flower buds of walnut. Among them, the expression characteristics of six JrTALE genes (JrTALE4-5, JrTALE8-12, JrTALE14-15, JrTALE23-25, JrTALE36-37 and JrTALE46-53) in flower buds were confirmed by qRT-PCR measurement. Overall, this study provides valuable information about the gene function of the TALE family in the transitional development of walnut flower buds, and the differential expression in male and female flower buds, which laid the foundation for future functional research.

\section{Methods}

\section{Plant materials}

All samples of this study were collected from walnut (Juglans regia L.), which trees were grown under natural conditions in the southern part of the Xinjiang Uyghur Autonomous Region, China.. male and female flower buds were collected before, during, after the flower transition period (FB / MB-1, FB / MB-2, and FB / MB-3). The samples were sealed with tin foil, immediately frozen in liquid nitrogen and stored at $-80^{\circ} \mathrm{C}$.

Page 5/18 


\section{Indentification of TALE family members in walnut}

Downloaded the latest protein sequence file (GCF_001411555.1_wgs.5d_protein.faa.gz) of the walnut genome from the NCBI website(ftp://ftp.ncbi.nlm.nih.gov/genomes/all/GCF/001/411/555/GCF_001411555.1_wgs.5d/GCF_001411555.1_wgs.5d_protein.faa.gz). The hidden Markov models (HMM) family (PF07526, PF05920, PF03790, PF03791, PF03789) were obtained from the Pfam online (http://pfam.xfam.org/) [47]. Based on hmmer3.0, the HMM model was used to retrieve the protein sequence of walnut [48-49] (with a threshold value of $E \leq 1 \mathrm{e}-20$ ), redundant sequences were removed, and the TALE gene family of walnut was identified initially. Then the candidate TALE members were uploaded to the CDD (https://www.ncbi.nlm.nih.gov/Structure/cdd/wrpsb.cgi) and SMART [50] (http://smart.embl-heidelberg.de/) online websites to further confirm that if they contained the proper TALE domain (remove sequences without TALE domain). The sequence was submitted to the ProtParam (https://www.expasy.org/protparam/) tool to predict its protein information, such as amino acid length, molecular weight, isoelectric point, and so on. the subcellular localization information of JrTALE protein was predicted by the online software ProtComp 9.0 (http://linux1.softberry.com/berry.phtml?

topic=protcomppl\&group=programs\&subgroup=proloc).

\section{Gene structure analysis and conserved motif}

The TALE gene structure information was extracted from the walnut genome information, and its exon and intron structure were analyzed using GSDS (http://gsds.cbi.pku.edu.cn/). The TBtools software's BioSequence Structure Illustrator program was used for drawing [51]. MEME program [52] (http://meme-suite.org/tools/meme) was used to identify the conserved motifs of JrTALE family members with a maximum number of motifs was 15 , other parameters were default.

\section{Phylogenetic analysis of TALE members}

The domain sequence of the TALE protein was downloaded from PlantTFDB (http://planttfdb.cbi.pku.edu.cn/) for walnuts, grapes and Arabidopsis. These sequences were aligned using MUSCLE, and then the neighbor-joining method of MEGA 6.0 software was used for phylogenetic analysis with 1000 bootstrap replicates.

\section{Cis-element analysis in the promoter of JrTALE gene}

The JrTALE gene sequence was submitted to the NCBI website and compared with the walnut genome sequence using the BLASTN program. The $2000 \mathrm{bp}$ sequence upstream of the gene was extracted as the promoter sequence of the JrTALE gene, and then the sequence was submitted to the online software PlantCARE (http://bioinformatics.psb.ugent.be/webtools/plantcare/html/) database to predict the promoter cis-acting element. the predicted results were visualized using TBtools software [51].

\section{Expression pattern analysis of JrTALE}

To analyze the expression pattern of JrTALE gene, we used RNA-seq data of Biomaker company to analyze the expression of walnut in different tissue parts (female flower buds and male flower buds) and different developmental stages (FB / MB-1, FB / MB-2, FB / MB-3). The FPKM values (Additional file 3: Table S2) were normalized by log10 (FPKM), which was visualized using Heml1.0 [53] software to heatmap.

\section{RNA isolation and qRT-PCR analysis}

The male and female flower buds (FB-1, FB-2, FB-3, MB-1, MB-2, MB-3) were collected at different developmental stages. Total RNA was extracted using the EASY spin Plus Plant RNA Rapid Extraction Kit (China, Aidlab) as described by the manufacturer, and Prime Script ${ }^{\mathrm{TM}}$ RT reagent Kit with gDNA Eraser Kit (TaKaRa, China) was reverse-transcribed into cDNA. The SYBR Green Prime Script RT-PCR Kit (Toyobo, Osaka, Japan) was used for qRT-PCR amplification in a CFX Connect ${ }^{\text {TM }}$ real-time PCR system (BioRad, USA). Walnut 18S-rRNA gene (forward: 5'-GGTCAATCTTCTCGTTCCCTT-3' and reverse: 5'- TCGCATTTCGCTACGTTCTT - 3') was used as the normalizer. The reaction conditions for real-time PCR were follows: initiation with $95^{\circ} \mathrm{C}$ for $5 \mathrm{~min}$, followed by 40 cycles of denaturation at $94^{\circ} \mathrm{C}$ for $30 \mathrm{~s}, 30 \mathrm{~s}$ at $55^{\circ} \mathrm{C}$, and $30 \mathrm{~s}$ at $72^{\circ} \mathrm{C}$. A melting curve was included from 65 to $95^{\circ} \mathrm{C}$ to verify the specificity of the amplified product. The relative expression levels of candidate genes were determined using the $2^{-\Delta \Delta \mathrm{Ct}}$ method [54]. Each treatment consists of three technical replicates. The primers used in this analysis were listed in Additional file 4: Table S3.

\section{Analysis of JrTALE Protein Interaction Network}

Each walnut TALE protein matched a homologous Arabidopsis TALE protein with the highest score by the blastp program (Additional file 5: Table S4), and thirty-three Arabidopsis TALE proteins which were represent 69 walnut TALE proteins were submitted to the STRING website 
(https://string-db.org) the program ran with default parameters to predict protein-protein interactions. In addition to 33 input proteins, five predictive functional partners were used to build the network.

\section{Abbreviations}

KNOX

KNOTTED-like homeodomain

BLH / BELL

BEL1-like homeodomain

STM

shoot meristemless

UTR

untranslated regions

FB-1 / FB-2 / FB-3

before, during, and after transformation of female flower buds

MB-1 / MB-2 / MB-3

before, during, and after transformation of male flower buds

qRT-PCR

quantitative real-time PCR

\section{Declarations}

\section{Acknowledgments}

Not applicable.

\section{Funding}

This work was supported by the important National Science and Technology Specific projects of Xinjiang (No.201130102-1-4) and the National Natural Science Foundation of China (No. 30560090). The funding institution was not involved in the design of the study, collection, analysis, and interpretation of data, and in writing the manuscript.

\section{Availability of data and materials}

Data generated or analyzed during this study are included in this article and its supplementary information files.

\section{Authors' contributions}

JXN and CHG designed the research. The materials were collected by CHG and SWQ, and RNA was extracted together with CK and JML. $\mathrm{CHG}$ and ZRZ conducted the bioinformatics analysis. $\mathrm{GCH}$ wrote the manuscript. All authors have read and approved the final manuscript.

\section{Ehtics approval and consent to participle}

Not applicable.

\section{Consent for publication}

Not applicable.

\section{Competing interests}

The authors declare that they have no competing interests.

\section{References}

1. Bürglin TR. Analysis of TALE superclass homeobox genes (MEIS, PBC, KNOX, Iroquois, TGIF) reveals a novel domain conserved between plants and animals. Nucleic acids research. 1997;25(21):4173-80.

2. Gehring WJ. Homeoboxes in the study of development. Science. 1987;236(4806):1245-52. doi:10.1126/science.2884726. 
3. Burglin TR. The PBC domain contains a MEINOX domain: coevolution of Hox and TALE homeobox genes? Dev Genes Evol. 1998;208(2):113-6. doi:10.1007/s004270050161.

4. Chen H, Rosin FM, Prat S, Hannapel DJ. Interacting transcription factors from the three-amino acid loop extension superclass regulate tuber formation. Plant physiology. 2003;132(3):1391-404.

5. Hamant O, Pautot V. Plant development: a TALE story. Comptes rendus biologies. 2010;333(4):371-81.

6. Smith HM, Hake S. The interaction of two homeobox genes, BREVIPEDICELLUS and PENNYWISE, regulates internode patterning in the Arabidopsis inflorescence. Plant Cell. 2003;15(8):1717-27.

7. Mukherjee K, Brocchieri L, Burglin TR. A comprehensive classification and evolutionary analysis of plant homeobox genes. Molecular biology evolution. 2009;26(12):2775-94.

8. Bharathan G, Janssen BJ, Kellogg EA, Sinha N. Phylogenetic relationships and evolution of the KNOTTED class of plant homeodomain proteins. Molecular biology and evolution 1999, 16(4):553-563.

9. Finn RD, Coggill P, Eberhardt RY, Eddy SR, Mistry J, Mitchell AL, Potter SC, Punta M, Qureshi M, Sangrador-Vegas A, et al. The Pfam protein family's database: towards a more sustainable future. Nucleic acids research. 2016;44(D1):D279-85.

10. Vollbrecht E, Veit B, Sinha N, Hake S. The developmental gene Knotted-1 Is a member of a maize homeobox gene family. Nature. 1991;350(6315):241-3.

11. Rupp HM, Frank M, Werner T, Strnad M, Schmulling T. Increased steady state mRNA levels of the STM and KNAT1 homeobox genes in cytokinin overproducing Arabidopsis thaliana indicate a role for cytokinins in the shoot apical meristem. The Plant journal: for cell molecular biology. 1999;18(5):557-63.

12. Endrizzi K, Moussian B, Haecker A, Levin JZ, Laux T. The SHOOT MERISTEMLESS gene is required for maintenance of undifferentiated cells in Arabidopsis shoot and floral meristems and acts at a different regulatory level than the meristem genes WUSCHEL and ZWILLE. The Plant journal: for cell molecular biology. 1996;10(6):967-79.

13. Li E, Bhargava A, Qiang W, Friedmann MC, Forneris N, Savidge RA, Johnson LA, Mansfield SD, Ellis BE, Douglas CJ. The Class II KNOX gene KNAT7 negatively regulates secondary wall formation in Arabidopsis and is functionally conserved in Populus. New Phytol. 2012;194(1):102-15.

14. Gong SY, Huang GQ, Sun X, Qin LX, Li Y, Zhou L, Li XB. Cotton KNL1, encoding a class II KNOX transcription factor, is involved in regulation of fibre development. J Exp Bot. 2014;65(15):4133-47.

15. Shani E, Yanai O, Ori N. The role of hormones in shoot apical meristem function. Curr Opin Plant Biol. 2006;9(5):484-9.

16. Hake S, Smith HM, Holtan H, Magnani E, Mele G, Ramirez J. The role of knox genes in plant development. Annu Rev Cell Dev Biol. 2004;20:125-51.

17. Qi B, Zheng H. Modulation of root-skewing responses by KNAT1 in Arabidopsis thaliana. The Plant journal: for cell molecular biology. 2013;76(3):380-92.

18. Wang S, Yamaguchi M, Grienenberger E, Martone PT, Samuels AL, Mansfield SD. The Class II KNOX genes KNAT3 and KNAT7 work cooperatively to influence deposition of secondary cell walls that provide mechanical support to Arabidopsis stems. The Plant journal: for cell molecular biology. 2020;101(2):293-309.

19. Furumizu C, Alvarez Jp, Sakakibara K, Bowman JL. Antagonistic roles for KNOX1 and KNOX2 genes in patterning the land plant body plan following an ancient gene duplication. PLoS Genet. 2015;11(2):e1004980.

20. Truernit E, Haseloff J. A Role for KNAT Class II Genes in Root Development. Plant Signal Behav. 2007;2(1):10-2.

21. Hay A, Tsiantis M. KNOX genes: versatile regulators of plant development and diversity. Development. 2010;137(19):3153-65.

22. Becker A, Bey M, Burglin TR, Saedler H, Theissen G. Ancestry and diversity of BEL1-like homeobox genes revealed by gymnosperm (Gnetum gnemon) homologs. Dev Genes Evol. 2002;212(9):452-7.

23. Bellaoui M, Pidkowich MS, Samach A, Kushalappa K, Kohalmi SE, Modrusan Z, Crosby WL, Haughn GW. The Arabidopsis BELL1 and KNOX TALE homeodomain proteins interact through a domain conserved between plants and animals. Plant Cell. 2001;13(11):245570.

24. Luo H, Song F, Goodman RM, Zheng Z. Up-regulation of OsBIHD1, a rice gene encoding BELL homeodomain transcriptional factor, in disease resistance responses. Plant biology (Stuttgart, Germany) 2005, 7(5):459-468.

25. Zhang L, Zhang X, Ju H, Chen J, Wang S, Wang H, Zhao Y, Chang Y. Ovate family protein1 interaction with BLH3 regulates transition timing from vegetative to reproductive phase in Arabidopsis. Biochem Biophys Res Commun. 2016;470(3):492-7.

26. Smith HM, Boschke I, Hake S. Selective interaction of plant homeo-domain proteins mediates high DNA-binding affinity. Proc Natl Acad Sci USA. 2002;99(14):9579-84. 
27. Chen H, Banerjee AK, Hannapel DJ. The tandem complex of BEL and KNOX. cell and molecular biology 2004, 38(2):276-284.

28. Mason J. "Differential analysis of gene expression in Arabidopsis thaliana organ boundary mutants" (2019). Honors Theses. 1228. https://egrove.olemiss.edu/hon_thesis/1228.

29. Bhatt AM, Etchells JP, Canales C, Lagodienko A, Dickinson H. VAAMANA-a BEL1- like homeodomain protein, interacts with KNOX proteins BP and STM and regulates inflorescence stem growth in Arabidopsis. Gene. 2004;328:103-11.

30. Kim D, Cho Young-hyun, Ryu H, Kim Y, Kim Tae-Houn, Hwang I. BLH 1 and KNAT 3 modulate ABA responses during germination and early seedling development in Arabidopsis. The Plant journal: for cell and molecular biology. 2013, 76(5).

31. Dinneny JR, Weigel D, Yanofsky MF. A genetic framework for fruit patterning in Arabidopsis thaliana. Development. 2005;132(21):4687-96.

32. Balanza V, Navarrete M, Trigueros M, Ferrandiz C. Patterning the female side of Arabidopsis: the importance of hormones. J Exp Bot. 2006;57(13):3457-69.

33. Pollegioni P, Woeste K, Chiocchini F, Del Lungo S, Ciolfi M, Olimpieri I, Tortolano V, Clark J, Hemery GE, Mapelli S, et al. Rethinking the history of common walnut (Juglans regia L.) in Europe: Its origins and human interactions. PloS one. 2017;12(3):e0172541.

34. Belles-Boix E, Hamant O, Witiak SM, Morin H, Traas J, Pautot V. KNAT6: an Arabidopsis homeobox gene involved in meristem activity and organ separation. Plant Cell. 2006;18(8):1900-7.

35. Ratcliffe OJ, Riechmann JL. Arabidopsis transcription factors and the regulation of flowering time: a genomic perspective. Curr Issues Mol Biol. 2002;4(3):77-91.

36. Cole M, Nolte C, Werr W. Nuclear import of the transcription factor SHOOTMERISTEMLESS depends on heterodimerization with BLH proteins expressed in discrete sub-domains of the shoot apical meristem of Arabidopsis thaliana. Nucleic acids research. 2006;34(4):1281-92.

37. Arnaud N, Pautot V. Ring the BELL and tie the KNOX: roles for TALEs in gynoecium development. Frontiers in plant science. $2014 ; 5: 93$.

38. Scofield S, Dewitte W, Murray JA. The KNOX gene SHOOT MERISTEMLESS is required for the development of reproductive meristematic tissues in Arabidopsis. The Plant journal: for cell molecular biology. 2007;50(5):767-81.

39. Khan M, Ragni L, Tabb P, Salasini BC, Chatfield S, Datla R, Lock J, Kuai X, Després C, Proveniers M, et al. Repression of Lateral Organ Boundary Genes by PENNYWISE and POUND-FOOLISH Is Essential for Meristem Maintenance and Flowering in Arabidopsis. Plant physiology. 2015;169(3):2166-86.

40. Ung N, Lal S, Smith HM. The role of PENNYWISE and POUND-FOOLISH in the maintenance of the shoot apical meristem in Arabidopsis. Plant physiology. 2011;156(2):605-14.

41. Kim D, Cho YH, Ryu H, Kim Y, Kim TH, Hwang I. BLH1 and KNAT3 modulate ABA responses during germination and early seedling development in Arabidopsis. The Plant journal: for cell molecular biology. 2013;75(5):755-66.

42. Hackbusch J, Richter K, Müller J, Salamini F, Uhrig JF. A central role of Arabidopsis thaliana ovate family proteins in networking and subcellular localization of 3-aa loop extension homeodomain proteins. Proc Natl Acad Sci USA. 2005;102(13):4908-12.

43. Rutjens B, Bao D, van Eck-Stouten E, Brand M, Smeekens S, Proveniers M. Shoot apical meristem function in Arabidopsis requires the combined activities of three BEL1-like homeodomain proteins. The Plant journal: for cell molecular biology. 2009;58(4):641-54.

44. Li Y, Pi L, Huang H, Xu L. ATH1 and KNAT2 proteins act together in regulation of plant inflorescence architecture. J Exp Bot. 2012;63(3):1423-33.

45. Alonso-Cantabrana H, Ripoll JJ, Ochando I, Vera A, Ferrándiz C, Martínez-Laborda A. Common regulatory networks in leaf and fruit patterning revealed by mutations in the Arabidopsis ASYMMETRIC LEAVES1 gene. Development. 2007;134(14):2663-71.

46. Guo M, Thomas J, Collins G, Timmermans MC. Direct repression of KNOX loci by the ASYMMETRIC LEAVES1 complex of Arabidopsis. Plant Cell. 2008;20(1):48-58.

47. El-Gebali S, Mistry J, Bateman A, Eddy SR, Luciani A, Potter SC, Qureshi M, Richardson LJ, Salazar GA, Smart A, et al. The Pfam protein families database in 2019. Nucleic acids research. 2019;47(D1):D427-d432.

48. Eddy SR. Accelerated Profile HMM Searches. PLoS Comput Biol. 2011;7(10):e1002195.

49. Soding J. Protein homology detection by HMM-HMM comparison. Bioinformatics. 2005;21(7):951-60.

50. Letunic I, Bork P. 20 years of the SMART protein domain annotation resource. Nucleic acids research. 2018;46(D1):D493-d496.

51. Chen CJ, Xia R, Hao C, He YH,2018, TBtools, a Toolkit for Biologists integrating various HTS-data hangding tools with a user-friendly interface. BioRxiv 2018.

52. Bailey TL, Boden M, Buske FA, Frith M, Grant CE, Clementi L, Ren J, Li WW. Noble WS: MEME SUITE: tools for motif discovery and searching. Nucleic acids research 2009, 37(Web Server issue): W202-208.

Page 9/18 
53. Deng W, Wang Y, Liu Z, Cheng H, Xue Y. Heml: a toolkit for illustrating heatmaps. PloS one 2014, 9(11): e111988.

54. Livak KJ, Schmittgen TD. Analysis of relative gene expression data using real-time quantitative PCR and the 2(-Delta Delta C(T)) Method. Methods. 2001;25(4):402-8.

\section{Tables}

Table 1 Characterization of the TALE gene family members in walnut. 


\begin{tabular}{|c|c|c|c|c|c|c|c|c|}
\hline Gene name & Gene symbol & Scaff & Scaff rename & Related protein & short name & $\begin{array}{l}\text { AA } \\
\text { Daal }\end{array}$ & MVIDaI & IP \\
\hline \multirow[t]{2}{*}{ JrTALE1-2 } & LOC108979090 & NW_017438852.1 & Scaff13 & XP_018805223.1 & JrTALE1 & 319 & 36126.63 & 4.87 \\
\hline & & & & XP_018805224.1 & JrTALE2 & 319 & 36126.63 & 4.87 \\
\hline JrTALE3 & LOC108979965 & NW_017439513.1 & Scaff14 & XP_018806314.1 & JrTALE3 & 375 & 42712.52 & 5.8 \\
\hline \multirow[t]{2}{*}{ JrTALE4-5 } & LOC108980836 & NW_017440201.1 & Scaff15 & XP_018807392.1 & JrTALE4 & 685 & 75261.69 & 6.13 \\
\hline & & & & XP_018807393.1 & JrTALE5 & 685 & 75261.69 & 6.13 \\
\hline \multirow[t]{2}{*}{ JrTALE6-7 } & LOC108981545 & NW_017440575.1 & Scaff16 & XP_018808307.1 & JrTALE6 & 356 & 40326.13 & 5.97 \\
\hline & & & & XP_018808309.1 & JrTALE7 & 356 & 40326.13 & 5.97 \\
\hline \multirow[t]{5}{*}{ JrTALE8-12 } & LOC108982860 & NW_017441761.1 & Scaff17 & XP_018809867.1 & JrTALE8 & 696 & 75926.63 & 6.91 \\
\hline & & & & XP_018809868.1 & JrTALE9 & 696 & 75926.63 & 6.91 \\
\hline & & & & XP_018809869.1 & JrTALE10 & 696 & 75926.63 & 6.91 \\
\hline & & & & XP_018809870.1 & JrTALE11 & 696 & 75926.63 & 6.91 \\
\hline & & & & XP_018809871.1 & JrTALE12 & 696 & 75926.63 & 6.91 \\
\hline JrTALE13 & LOC108986966 & NW_017442862.1 & Scaff18 & XP_018815324.1 & JrTALE13 & 682 & 74968.47 & 5.68 \\
\hline \multirow[t]{2}{*}{ JrTALE14-15 } & LOC108988270 & NW_017443011.1 & Scaff19 & XP_018817027.1 & JrTALE14 & 722 & 78843.08 & 6.65 \\
\hline & & & & XP_018817028.1 & JrTALE15 & 722 & 78843.08 & 6.65 \\
\hline \multirow[t]{7}{*}{ JrTALE16-22 } & LOC108990328 & NW_017443280.1 & Scaff20 & XP_018819801.1 & JrTALE16 & 850 & 93611.11 & 6.64 \\
\hline & & & & XP_018819802.1 & JrTALE17 & 850 & 93611.11 & 6.64 \\
\hline & & & & XP_018819803.1 & JrTALE18 & 850 & 93611.11 & 6.64 \\
\hline & & & & XP_018819804.1 & JrTALE19 & 850 & 93611.11 & 6.64 \\
\hline & & & & XP_018819805.1 & JrTALE20 & 850 & 93611.11 & 6.64 \\
\hline & & & & XP_018819806.1 & JrTALE21 & 850 & 93611.11 & 6.64 \\
\hline & & & & XP_018819807.1 & JrTALE22 & 850 & 93611.11 & 6.64 \\
\hline \multirow[t]{3}{*}{ JrTALE23-25 } & LOC108991317 & NW_017443495.1 & Scaff21 & XP_018821045.1 & JrTALE23 & 565 & 62686.78 & 5.89 \\
\hline & & & & XP_018821046.1 & JrTALE24 & 565 & 62686.78 & 5.89 \\
\hline & & & & XP_018821047.1 & JrTALE25 & 565 & 62686.78 & 5.89 \\
\hline \multirow[t]{2}{*}{ JrTALE26-27 } & LOC108992228 & NW_017443527.1 & Scaff22 & XP_018822263.1 & JrTALE26 & 477 & 53076.64 & 6.04 \\
\hline & & & & XP_018822264.1 & JrTALE27 & 476 & 52920.46 & 5.98 \\
\hline JrTALE28 & LOC108995343 & NW_0174435578.1 & Scaff23 & XP_018826438.1 & JrTALE28 & 824 & 89539.65 & 6.85 \\
\hline \multirow[t]{3}{*}{ JrTALE29-31 } & LOC108997442 & NW_017443603.1 & Scaff24 & XP_018829277.1 & JrTALE29 & 676 & 75710.95 & 6.01 \\
\hline & & & & XP_018829278.1 & JrTALE30 & 676 & 75710.95 & 6.01 \\
\hline & & & & XP_018829279.1 & JrTALE31 & 676 & 75710.95 & 6.01 \\
\hline \multirow[t]{2}{*}{ JrTALE32-33 } & LOC108998188 & NW_017443616.1 & Scaff25 & XP_018830219.1 & JrTALE32 & 846 & 91749.98 & 6.6 \\
\hline & & & & XP_018830220.1 & JrTALE33 & 846 & 91749.98 & 6.6 \\
\hline JrTALE34 & LOC109001756 & NW_017388815.1 & Scaff1 & XP_018834699.1 & JrTALE34 & 321 & 36251.17 & 5.24 \\
\hline JrTALE35 & LOC109003871 & NW_017389148.1 & Scaff2 & XP_018837747.1 & JrTALE35 & 322 & 36365.87 & 5 \\
\hline \multirow[t]{2}{*}{ JrTALE36-37 } & LOC109008224 & NW_017389472.1 & Scaff3 & XP_018843781.1 & JrTALE36 & 484 & 53514.16 & 5.92 \\
\hline & & & & XP_018843782.1 & JrTALE37 & 483 & 53357.97 & 5.86 \\
\hline
\end{tabular}




\begin{tabular}{|lllllllll|}
\hline JrTALE38-39 & LOC109008282 & NW_017389474.1 & Scaff4 & XP_018843851.1 & JrTALE38 & 682 & 75108.49 & 5.5 \\
\cline { 5 - 9 } & & & & XP_018843852.1 & JrTALE39 & 606 & 66806.45 & 5.51 \\
\hline JrTALE40 & LOC109008283 & NW_017389474.1 & Scaff4 & XP_018843853.1 & JrTALE40 & 511 & 56856.81 & 5.92 \\
\hline
\end{tabular}

Table1 Characterization of the TALE gene family members in walnut (Continued)

\begin{tabular}{|c|c|c|c|c|c|c|c|c|}
\hline Gene name & Gene symbol & Scaff & $\begin{array}{l}\text { Scaff } \\
\text { rename }\end{array}$ & Related protein & $\begin{array}{l}\text { short } \\
\text { name }\end{array}$ & $\begin{array}{l}\text { AA } \\
\text { (aa) }\end{array}$ & MVaDal & IP \\
\hline JrTALE41 & LOC109008556 & NW_017389505.1 & Scaff5 & XP_018844242.1 & JrTALE41 & 349 & 39490.4 & 5.79 \\
\hline JrTALE42 & LOC109008843 & NW_017389545.1 & Scaff6 & XP_018844620.1 & JrTALE42 & 376 & 43255.05 & 5.87 \\
\hline \multirow[t]{2}{*}{ JrTALE43-44 } & LOC109008860 & NW_017389545.1 & Scaff6 & XP_018844647.1 & JrTALE43 & 583 & 65243.78 & 8.02 \\
\hline & & & & XP_018844648.1 & JrTALE44 & 583 & 65243.78 & 8.02 \\
\hline JrTALE45 & LOC109011646 & NW_017389777.1 & Scaff7 & XP_018848478.1 & JrTALE45 & 331 & 37747.19 & 6.35 \\
\hline \multirow[t]{8}{*}{ JrTALE46-53 } & LOC109013525 & NW_017389916.1 & Scaff8 & XP_018851194.1 & JrTALE46 & 697 & 76014.5 & 7.79 \\
\hline & & & & XP_018851195.1 & JrTALE47 & 697 & 76014.5 & 7.79 \\
\hline & & & & XP_018851196.1 & JrTALE48 & 697 & 76014.5 & 7.79 \\
\hline & & & & XP_018851197.1 & JrTALE49 & 697 & 76014.5 & 7.79 \\
\hline & & & & XP_018851198.1 & JrTALE50 & 697 & 76014.5 & 7.79 \\
\hline & & & & XP_018851199.1 & JrTALE51 & 697 & 76014.5 & 7.79 \\
\hline & & & & XP_018851200.1 & JrTALE52 & 697 & 76014.5 & 7.79 \\
\hline & & & & XP_018851201.1 & JrTALE53 & 697 & 76014.5 & 7.79 \\
\hline JrTALE54 & LOC109014498 & NW_017389990.1 & Scaff9 & XP_018852535.1 & JrTALE54 & 672 & 75268.55 & 6.52 \\
\hline JrTALE55 & LOC109017125 & NW_017416914.1 & Scaff10 & XP_018854978.1 & JrTALE55 & 251 & 28666.7 & 9.77 \\
\hline \multirow[t]{3}{*}{ JrTALE56-58 } & LOC109019501 & NW_017436672.1 & Scaff11 & XP_018857333.1 & JrTALE56 & 837 & 92709.23 & 6.65 \\
\hline & & & & XP_018857334.1 & JrTALE57 & 825 & 91487.85 & 6.77 \\
\hline & & & & XP_018857335.1 & JrTALE58 & 815 & 90290.68 & 6.9 \\
\hline \multirow[t]{11}{*}{ JrTALE59-69 } & LOC109021085 & NW_017437773.1 & Scaff12 & XP_018859168.1 & JrTALE59 & 590 & 65712.02 & 6.35 \\
\hline & & & & XP_018859169.1 & JrTALE60 & 590 & 65712.02 & 6.35 \\
\hline & & & & XP_018859170.1 & JrTALE61 & 590 & 65712.02 & 6.35 \\
\hline & & & & XP_018859171.1 & JrTALE62 & 590 & 65712.02 & 6.35 \\
\hline & & & & XP_018859172.1 & JrTALE63 & 590 & 65712.02 & 6.35 \\
\hline & & & & XP_018859173.1 & JrTALE64 & 590 & 65712.02 & 6.35 \\
\hline & & & & XP_018859174.1 & JrTALE65 & 590 & 65712.02 & 6.35 \\
\hline & & & & XP_018859175.1 & JrTALE66 & 590 & 65712.02 & 6.35 \\
\hline & & & & XP_018859176.1 & JrTALE67 & 590 & 65712.02 & 6.35 \\
\hline & & & & XP_018859177.1 & JrTALE68 & 590 & 65712.02 & 6.35 \\
\hline & & & & XP_018859178.1 & JrTALE69 & 590 & 65712.02 & 6.35 \\
\hline
\end{tabular}

NoteIAA: amino acid, MV: molecular weight, IP: iso-electric point 


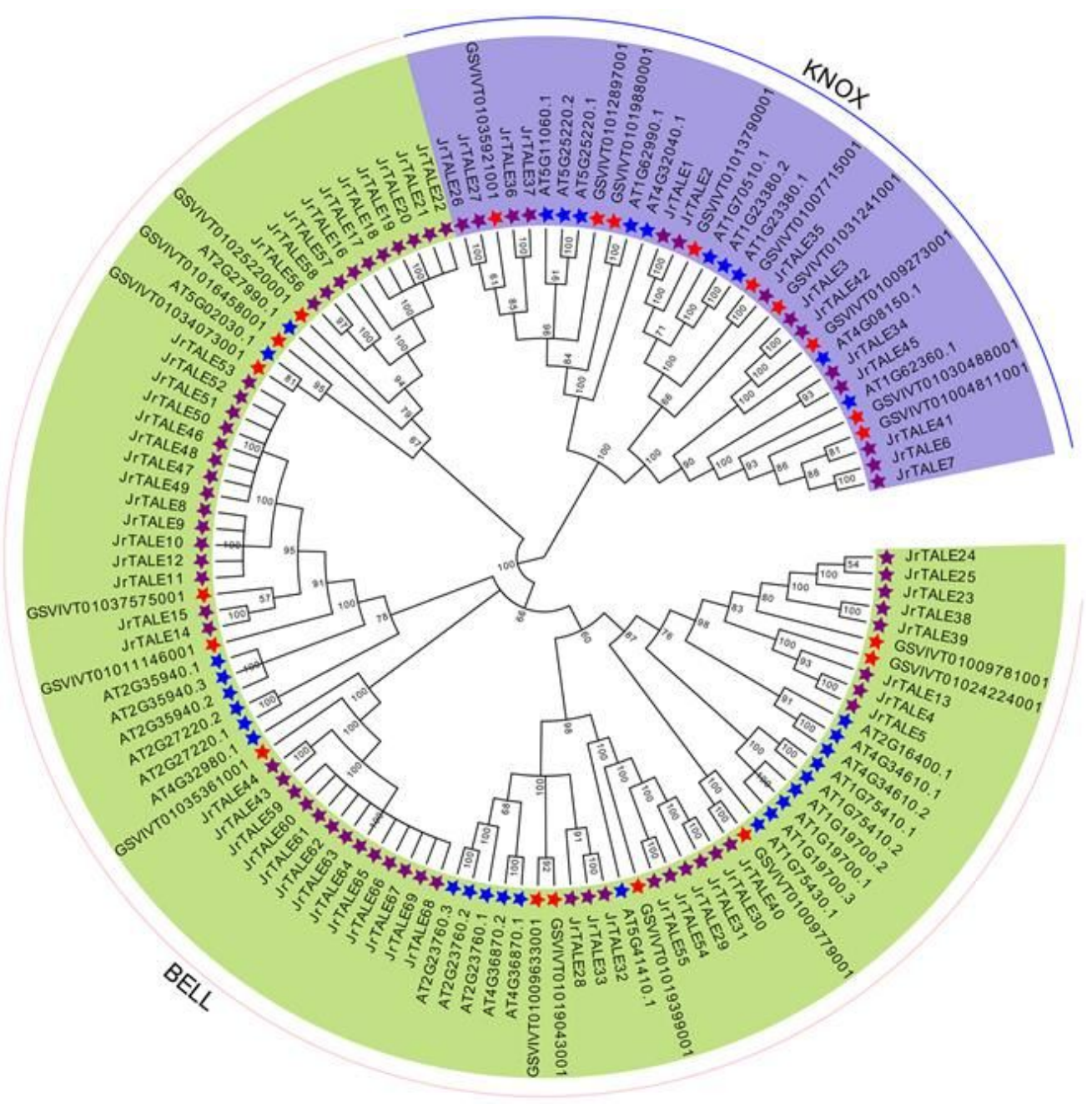

\section{Figure 1}

Phylogenetic analysis and classification of BELL and KNOX subfamily genes in walnut, Arabidopsis and grape. The phylogenetic tree was constructed by MEGA 6.0 software using the neighbor-joining $(\mathrm{NJ})$ method with 1000 bootstrap replicates. The genes for walnuts, Arabidopsis and grapes are marked with stars in purple, blue and red, respectively. 


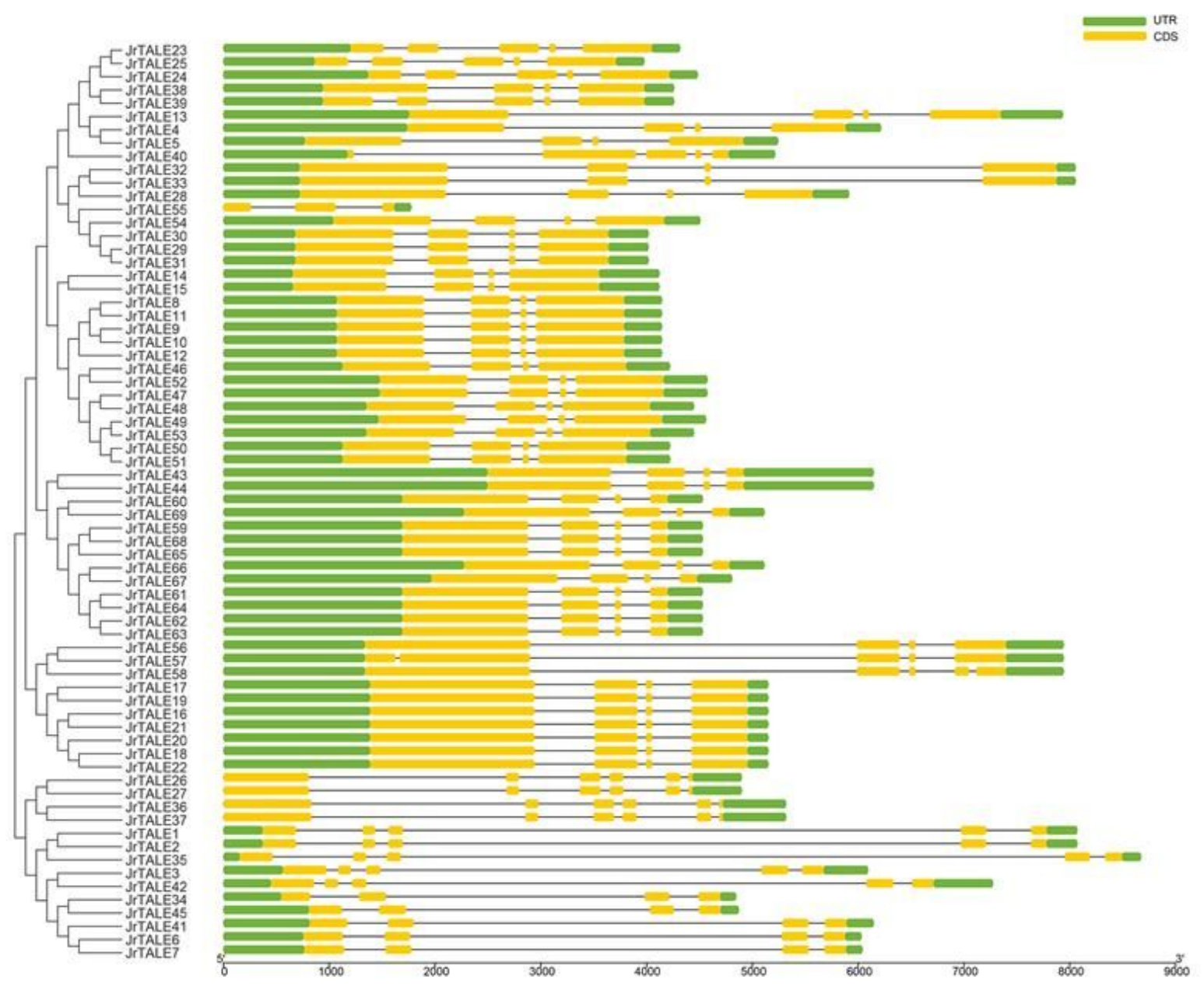

\section{Figure 2}

Gene structure analysis of JrTALE family gene. Phylogenetic relationship, Gene structure maps were drawn with the Gene Structure Display Server 2.0. The scale bar is shown at the bottom. 


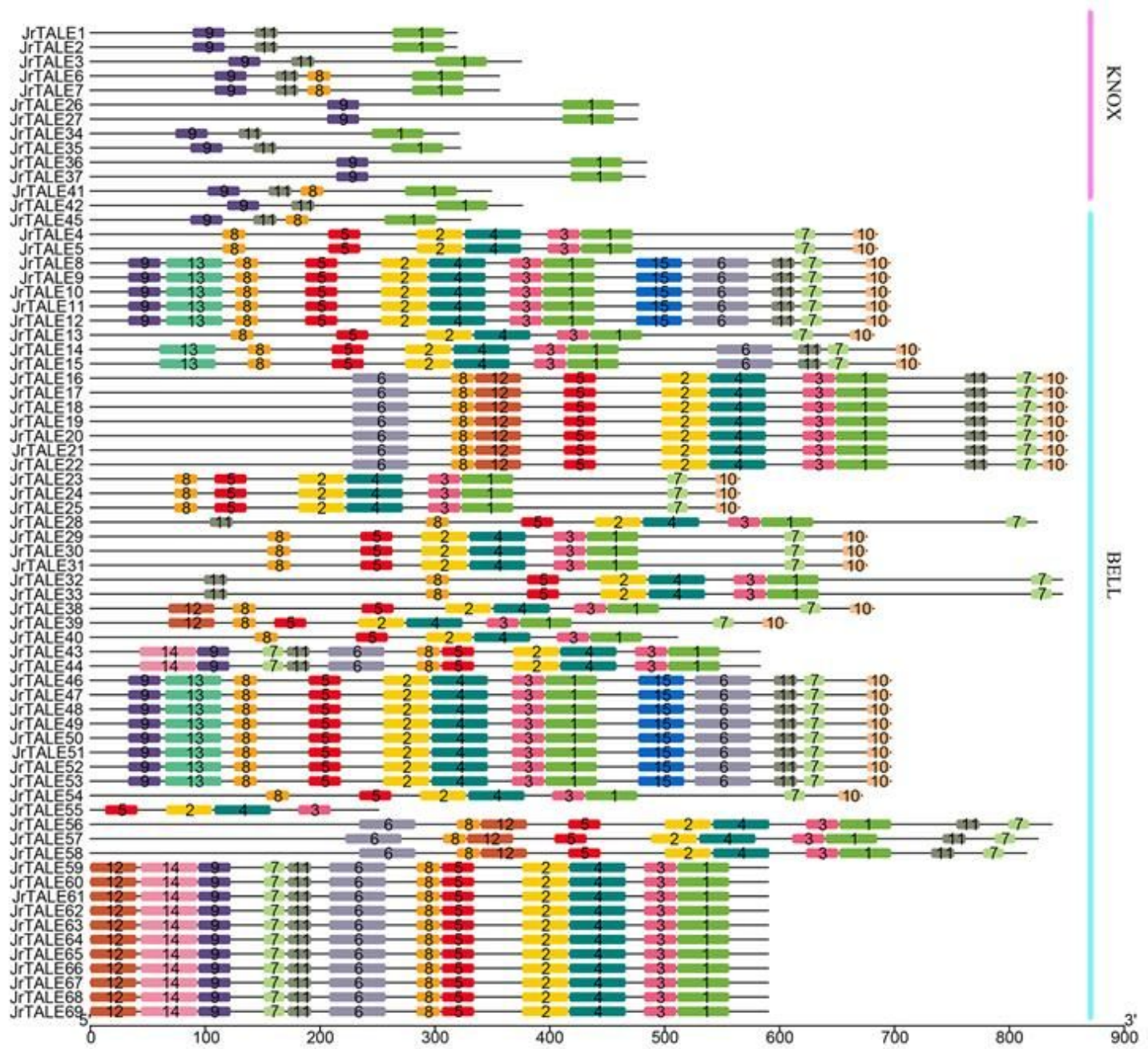

Figure 3

Architecture of conserved protein motifs of JrTALE family members by MEME analysis. 


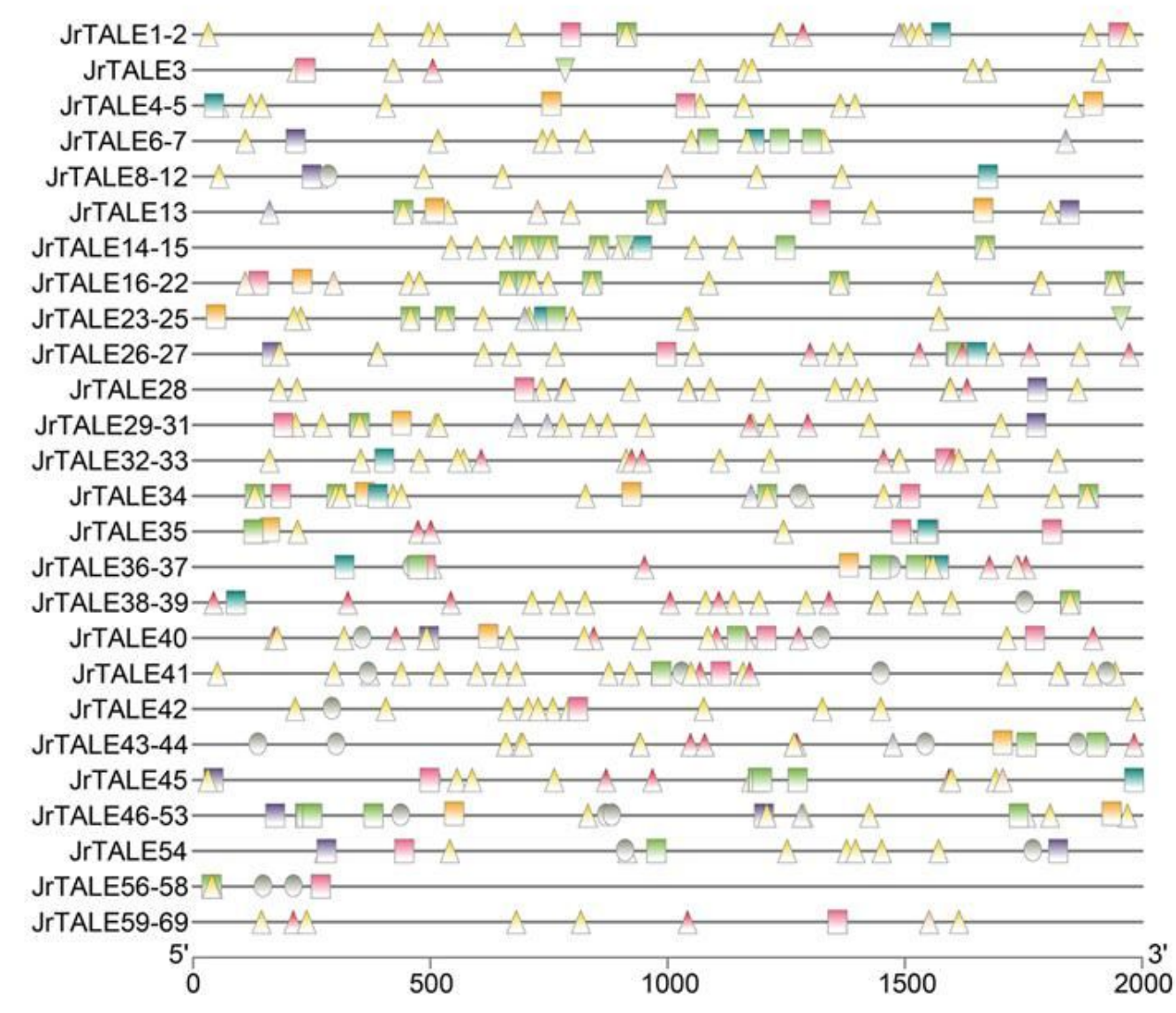

Abscisic acid responsive Light responsive

MeJA-responsive

Auxin-responsive

Anaerobic induction

Defense and stress

Drought-inducibility

Salicylic acid responsive

Gibberllin-responsive

Low-temperature responsive MYB binding site

\section{Figure 4}

Cis-element analysis of putative JrTALE promoters related to stress responses. Different cis-elements with the same or similar functions are shown in the same color and shape. The up-triangle, rectangle, and eclipse represented the environmental response, hormone responsive and myb-binding site, respectively.

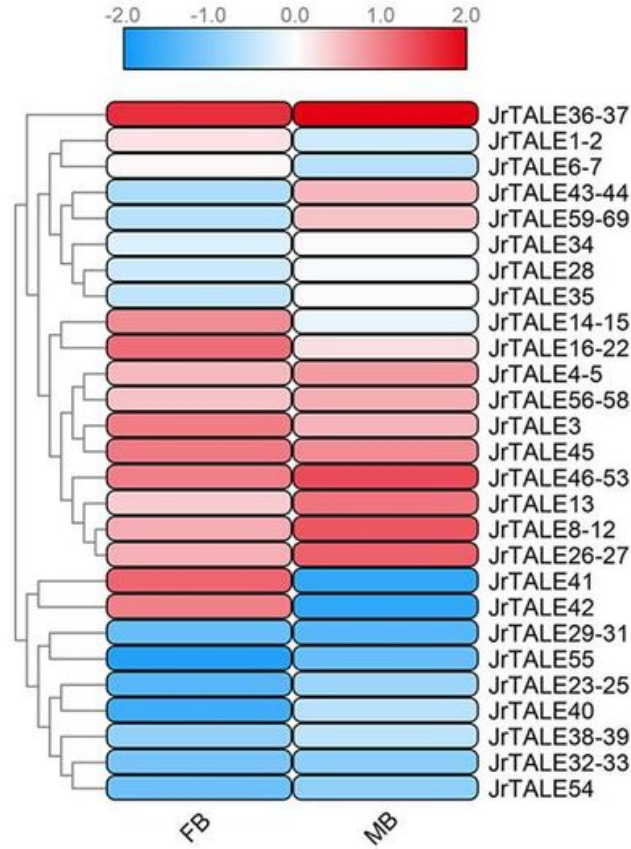

A

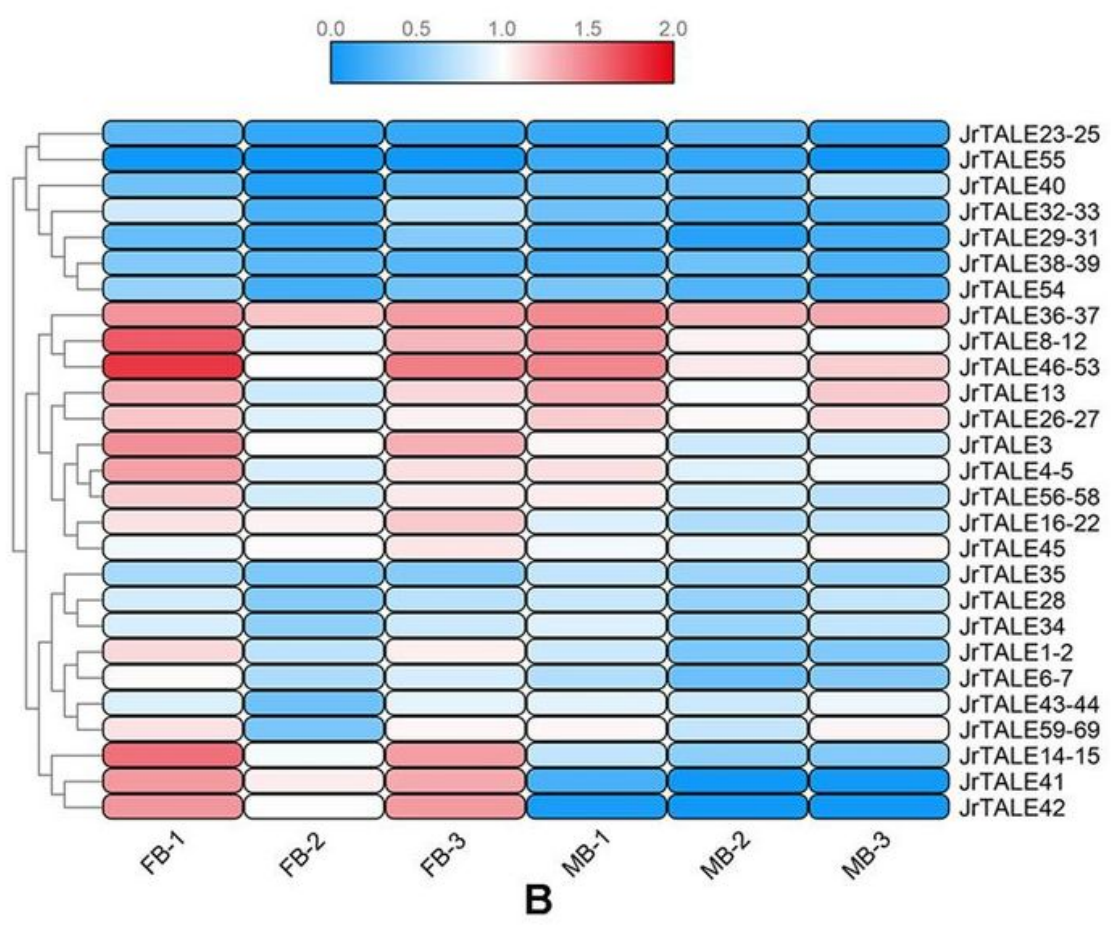

Page 16/18 


\section{Figure 5}

Expression profiles of candidate JrTALE genes in tissues or different developmental stages. A. Heatmap of the JrTALE genes between female flower buds and male flower buds. B. Heatmap of JrTALE genes expressed differently in three development periods of female and male flower buds (FB/MB-1, FB/MB-2, and FB/MB-3).
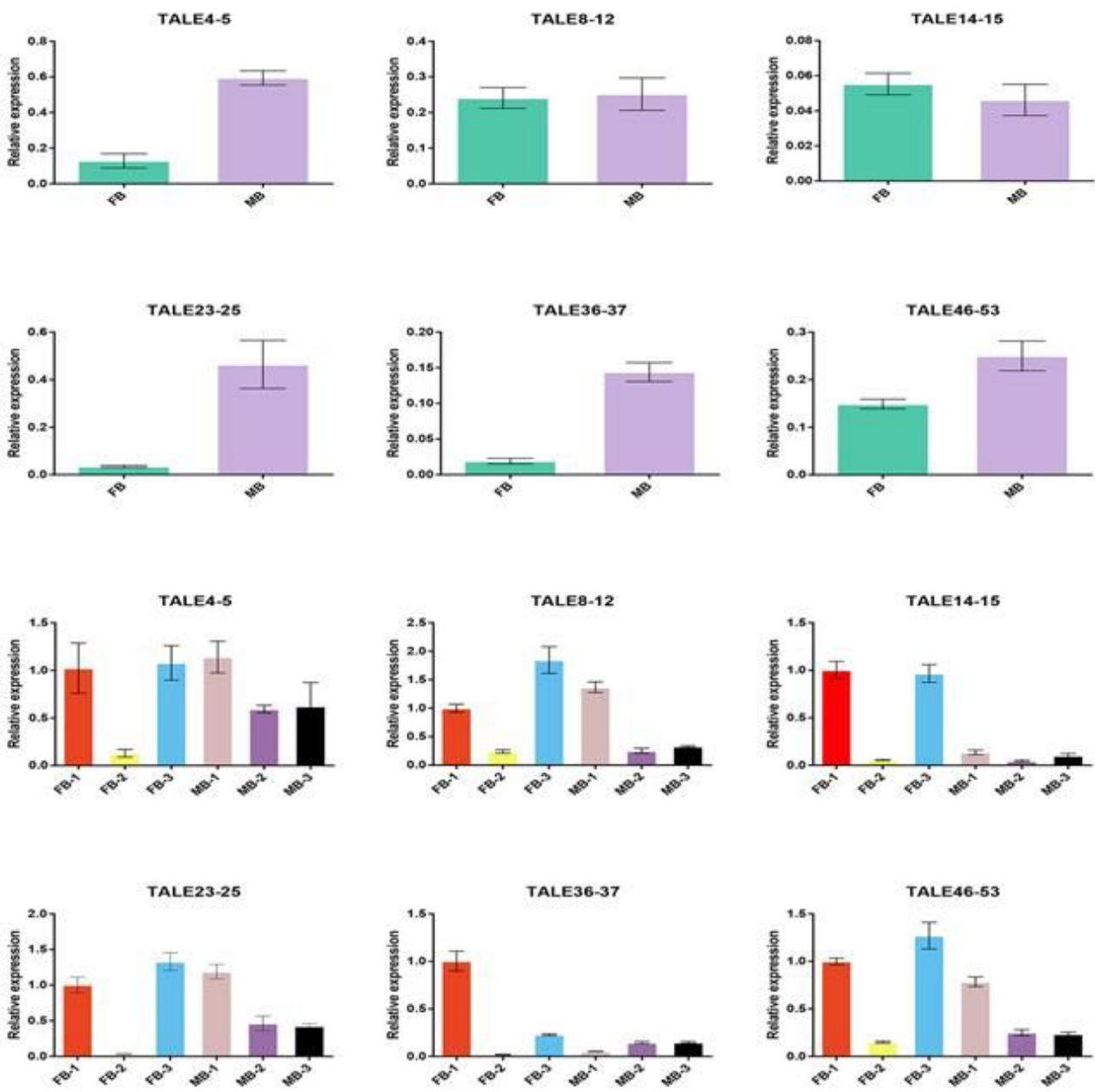

Figure 6

qRT-PCR analysis of JrTALE genes in different tissues and different development period of flower buds. 


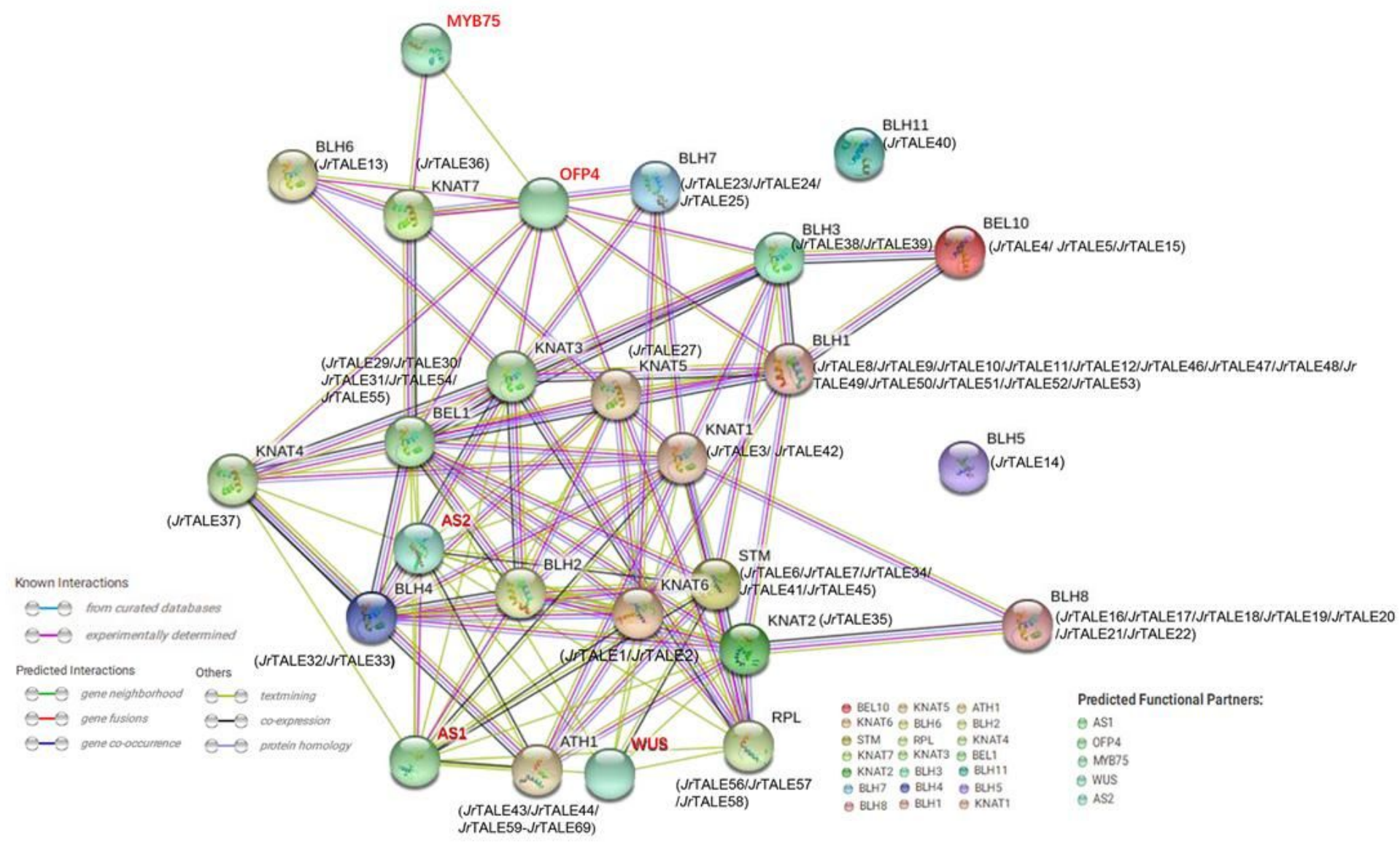

\section{Figure 7}

Predicted protein-protein interaction network of JrTALE protein. The network nodes represent proteins. The 3D structure of the protein is shown inside the nodes, and the line color indicates the type of interaction evidence. the network was constructed using sixteen input proteins (BEL1, BEL10, BLH1, BLH2, BLH3, BLH4, BLH5, BLH6, BLH7, BLH8, BLH11, ATH1, RPL, KNAT1, KNAT2, KNAT3, KNAT4, KNAT5, KNAT6, KNAT7 and STM ) and five predicted functional partners (AS1, AS2, OFP4, MYB75 and WUS).

\section{Supplementary Files}

This is a list of supplementary files associated with this preprint. Click to download.

- Additionalfile5.doc

- Additionalfile4.doc

- Additionalfile3.doc

- Additionalfile2.doc

- Additionalfile1.pdf 